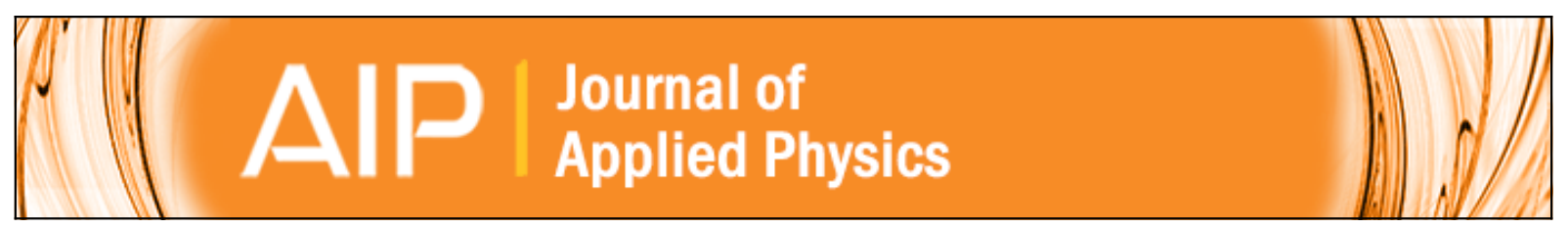

\title{
Magnetic properties of nearly stoichiometric CeAuBi2 heavy fermion compound
}

C. Adriano, P. F. S. Rosa, C. B. R. Jesus, T. Grant, Z. Fisk, D. J. Garcia, and P. G. Pagliuso

Citation: Journal of Applied Physics 117, 17C103 (2015); doi: 10.1063/1.4906279

View online: http://dx.doi.org/10.1063/1.4906279

View Table of Contents: http://scitation.aip.org/content/aip/journal/jap/117/17?ver=pdfcov

Published by the AIP Publishing

\section{Articles you may be interested in}

The effect of $\mathrm{Au}$ and $\mathrm{Ni}$ doping on the heavy fermion state of the Kondo lattice antiferromagnet CePtZn

J. Appl. Phys. 115, 17E113 (2014); 10.1063/1.4862378

Evolution of the magnetic properties along the $\mathrm{RCuBi} 2(\mathrm{R}=\mathrm{Ce}, \mathrm{Pr}, \mathrm{Nd}, \mathrm{Gd}, \mathrm{Sm})$ series of intermetallic compounds

J. Appl. Phys. 115, 17E115 (2014); 10.1063/1.4860657

Complex magnetic states of the heavy fermion compound CeGe

Low Temp. Phys. 38, 651 (2012); 10.1063/1.4734012

Magnetic properties of heavy fermion system Ce $1-x$ Gd x CoSi 3

J. Appl. Phys. 105, 07E122 (2009); 10.1063/1.3076604

Pressure and magnetic field effects In CeNiGe 2

J. Appl. Phys. 93, 8343 (2003); 10.1063/1.1556277

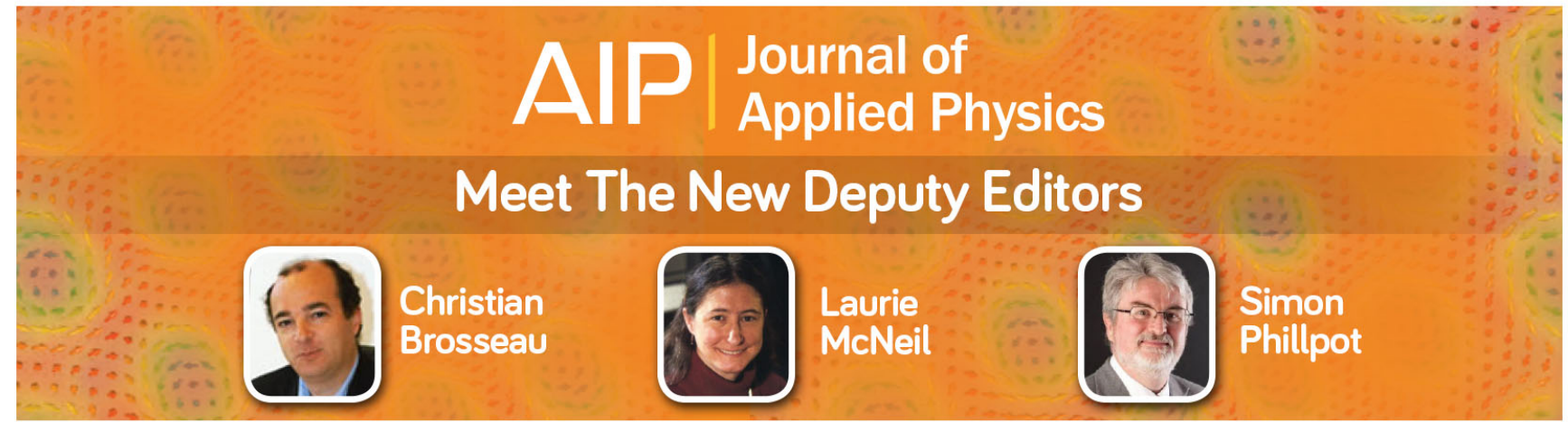




\title{
Magnetic properties of nearly stoichiometric $\mathrm{CeAuBi}$ heavy fermion compound
}

\author{
C. Adriano, ${ }^{1, a)}$ P. F. S. Rosa,,${ }^{1,2}$ C. B. R. Jesus, ${ }^{1}$ T. Grant, ${ }^{2}$ Z. Fisk, ${ }^{2}$ D. J. Garcia, ${ }^{3}$ \\ and P. G. Pagliuso ${ }^{1}$ \\ ${ }^{1}$ Instituto de Física “Gleb Wataghin,” UNICAMP, Campinas-SP, 13083-859, Brazil \\ ${ }^{2}$ University of California, Irvine, California 92697-4574, USA \\ ${ }^{3}$ Instituto Balseiro, Centro Atomico Bariloche, CNEA and CONICET, 8400 Bariloche, Argentina
}

(Presented 6 November 2014; received 22 September 2014; accepted 1 October 2014; published online 21 January 2015)

\begin{abstract}
Motivated by the interesting magnetic anisotropy found in the heavy fermion family $\mathrm{Ce}_{2} \mathrm{X}_{2}$ ( $T=$ transition metal and $X=$ pnictogen), here, we study the novel parent compound $\mathrm{CeAu}_{1-x} \mathrm{Bi}_{2-y}$ by combining magnetization, pressure dependent electrical resistivity, and heat-capacity measurements. The magnetic properties of our nearly stoichiometric single crystal sample of $\mathrm{CeAu}_{1-x} \mathrm{Bi}_{2-y}(x=0.92$ and $y=1.6)$ revealed an antiferromagnetic ordering at $T_{\mathrm{N}}=12 \mathrm{~K}$ with an easy axis along the $c$-direction. The field dependent magnetization data at low temperatures reveal the existence of a spin-flop transition when the field is applied along the $c$-axis $\left(H_{c} \sim 7.5 \mathrm{~T}\right.$ and $T=5 \mathrm{~K})$. The heat capacity and pressure dependent resistivity data suggest that $\mathrm{Ce} \mathrm{Au}_{0.92} \mathrm{Bi}_{1.6}$ exhibits a weak heavy fermion behavior with strongly localized $\mathrm{Ce}^{3+} 4 f$ electrons. Furthermore, the systematic analysis using a mean field model including anisotropic nearest-neighbors interactions and the tetragonal crystalline electric field (CEF) Hamiltonian allows us to extract a CEF scheme and two different values for the anisotropic $J_{\mathrm{RKKY}}$ exchange parameters between the $\mathrm{Ce}^{3+}$ ions in this compound. Thus, we discuss a scenario, considering both the anisotropic magnetic interactions and the tetragonal CEF effects, in the $\mathrm{CeAu}_{1-x} \mathrm{Bi}_{2-y}$ compounds, and we compare our results with the isostructural compound $\mathrm{CeCuBi}_{2}$. C 2015 AIP Publishing LLC.
\end{abstract}

[http://dx.doi.org/10.1063/1.4906279]

\section{INTRODUCTION}

The presence of Ruderman-Kittel-Kasuya-Yosida (RKKY) magnetic interactions, crystalline electrical field (CEF) effects and the hybridization between the $f$-electrons and the conduction electrons (the Kondo effect) is often responsible for the interesting variety of physical properties that can be found in Ce-based strongly correlated electron systems. ${ }^{1-3}$ For instance, $\mathrm{CeCoIn}_{5}$ displays quantum critical behavior, ${ }^{4,5} \mathrm{CeAgBi}_{2}$ presents complex magnetic ordering, ${ }^{6}$ and unconventional superconductivity can be found in $\mathrm{CeCu}_{2} \mathrm{Si}_{2}$ at ambient pressure and in $\mathrm{CeRhIn}_{5}$ under applied pressure. ${ }^{7,8}$ However, the understanding of the role of each one of these interactions and how the competition between them can determine the properties of a heavy fermion (HF) material is still one of the most challenging scientific problems. As such, the study of structurally related series of compounds represents a great opportunity to explore the evolution of the properties along its members.

In this context, here, we revisit the CeTX ${ }_{2}$ family ( $T=$ transition metal and $X=$ pnictogen) by studying and modeling the macroscopic properties of a novel member with $T=\mathrm{Au}$ and $X=\mathrm{Bi}$. Despite the recent attention given to the $\mathrm{Ce}_{\mathrm{BBi}}$ family $(T=\mathrm{Ni}, \mathrm{Cu}, \mathrm{Ag}$, and $\mathrm{Pd})$ of antiferromagnetic (AFM) compounds, a detailed analysis of the evolution of the relevant interactions in the series is missing. ${ }^{6,9-12} \mathrm{In}$

\footnotetext{
a) Author to whom correspondence should be addressed. Electronic mail: cadriano@ifi.unicamp.br.
}

particular, we have recently reported the physical properties and magnetic structure of the $\mathrm{CeCuBi}_{2}$ compound, ${ }^{13}$ which present an AFM ordering at $T_{\mathrm{N}}=16 \mathrm{~K}$. X-ray magnetic diffraction data below transition temperature $\left(T_{\mathrm{N}}\right)$ revealed a commensurate AFM structure with a propagation vector $\left(00 \frac{1}{2}\right)$ with the magnetic moments aligned along the $c$-axis, in agreement with the large magnetic anisotropy found in the susceptibility data. Furthermore, a mean field theory with the contribution of anisotropic first-neighbor interactions and tetragonal CEF (Ref. 14) was applied and allowed us to extract the CEF scheme for $\mathrm{CeCuBi}_{2}$. In this regard, the $T=$ Au member is a suitable choice to explore the evolution of the interactions since it is an isovalent substitution to the already known $T=\mathrm{Cu}, \mathrm{Ag}$ members.

$\mathrm{CeAuBi}_{2}$ crystallizes in the tetragonal $\mathrm{ZrCuSi}_{2}$-type structure $(P 4 / \mathrm{nmm}$ space group) with a stacking arrangement of $\mathrm{CeBi}-\mathrm{Au}-\mathrm{CeBi}-\mathrm{Bi}$ layers. In this work, we present the physical properties of $\mathrm{CeAu}_{0.92} \mathrm{Bi}_{1.6}$ studied by means of magnetic susceptibility, heat capacity, and electrical resistivity measurements. The same mean field theory discussed above, was applied to fit the magnetization data of this compound and the results show the evolution of the CEF scheme from $\mathrm{CeCuBi}_{2}$ to $\mathrm{CeAu}_{0.92} \mathrm{Bi}_{1.6}$.

\section{EXPERIMENTAL DETAILS}

Single crystals of $\mathrm{CeAu}_{0.92} \mathrm{Bi}_{1.6}$ were grown from Biflux with starting composition $\mathrm{Ce}: \mathrm{Au}: \mathrm{Bi}=1: 1: 20$. The sealed tube was heated up to $1050^{\circ} \mathrm{C}$ for $4 \mathrm{~h}$ and then cooled down 
at $5{ }^{\circ} \mathrm{C} / \mathrm{h}$. The excess of $\mathrm{Bi}$ flux was removed at $350^{\circ} \mathrm{C}$ by centrifugation and platelets-like crystals were mechanically removed from the crucible. ${ }^{15}$ The crystallographic structure was verified by X-ray powder diffraction and the extracted lattice parameters $(a=4.647(5) \AA$ and $c=9.901(5) \AA)$ are larger than the ones obtained for $\mathrm{CeCuBi}_{2}(a=4.555(4) \AA$ and $c=9.777(8) \AA$ ), as expected from the lattice expansion due to the larger transition metal ion. In addition, the sample was submitted to elemental analysis using a commercial Energy Dispersive Spectroscopy (EDS) microprobe and a commercial Wavelength Dispersive Spectroscopy (WDS), which revealed the stoichiometry to be 1:0.92:1.6 with an error of 5\%. Although only cleaved samples were used in our measurements, we attribute the Bi deficiency partially to the high air-sensitivity of the samples, which likely induces the formation of thin layers of $\mathrm{Bi}$ oxide on the surface.

Magnetization measurements were performed using a commercial superconducting quantum interference device (SQUID). The specific heat was measured using a commercial small mass calorimeter that employs a quasi-adiabatic thermal relaxation technique. The in-plane electrical resistivity measurements under applied pressure were carried out in a clamp-type $\mathrm{Cu}$-Be cell using Fluorinert as a pressure transmitting medium and a low-frequency ac resistance bridge.

\section{RESULTS AND DISCUSSIONS}

Figures 1(a) and 1(b) show the magnetic susceptibility $\chi(T)$ as a function of temperature for a magnetic field of 1 kOe applied parallel $\chi_{\|}$(panel 1(a)) and perpendicular $\chi_{\perp}$ (panel 1(b)) to the crystallographic $c$-axis. These data show an AFM order at $T_{\mathrm{N}} \simeq 12 \mathrm{~K}$ with an easy axis along the $c$ direction. The low temperature magnetic anisotropy can be revealed by calculating the ratio $\chi_{\|} / \chi_{\perp} \approx 17$ at $T_{\mathrm{N}}$ that is mainly determined by the tetragonal CEF splitting. Same analysis has been made for $\mathrm{CeCuBi}_{2}$ where it was found that
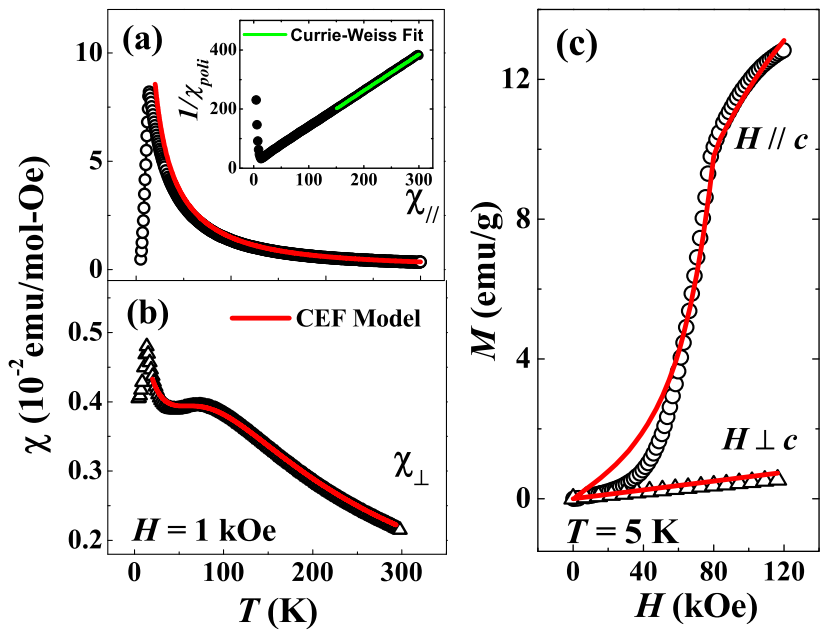

FIG. 1. Temperature dependence of the magnetic susceptibility measured with $H=1$ kOe applied (a) parallel $\chi_{\|}$and (b) perpendicular $\chi_{\perp}$ to the $c$-axis. (c) Magnetization as a function of the applied magnetic field parallel (open circles) and perpendicular (open triangles) to the $c$-axis at $T=5 \mathrm{~K}$. The inset on (a) shows the inverse of the polycrystalline average $1 / \chi_{\text {poly }}(T)$. The green-dashed line represents a Currie-Weiss fit for $T>150 \mathrm{~K}$. The redsolid lines through the experimental points in Figs. 1(a)-1(c) are best fits of the data using the CEF mean field model discussed in the text. $\chi_{\|} / \chi_{\perp} \approx 4.5$ at $T_{\mathrm{N}}$. The inverse of the polycrystalline $1 / \chi_{\text {poly }}(T)$ is presented in the inset of Fig. 1(a). A CurieWeiss fit for $T>150 \mathrm{~K}$ (dashed line) yields an effective magnetic moment $\mu_{\text {eff }}=2.55(2) \mu_{B}$ (in agreement with the theoretical value of $\mu_{\text {eff }}=2.54 \mu_{B}$ for $\mathrm{Ce}^{3+}$ ) and a paramagnetic Curie-Weiss temperature $\theta_{p}=-21 \mathrm{~K}$.

Figure 1(c) displays the field dependent magnetization $M(H)$ taken at $5 \mathrm{~K}$. A spin-flop transition from an antiferromagnetic to a ferromagnetic (FM) phase occurs at $H \approx 75 \mathrm{kOe}$ when the magnetic field is applied parallel to the $c$-axis (open circles). On the other hand, a linear behavior is observed for $H \perp c$-axis (open triangles) for fields up to $H=120 \mathrm{kOe}$, which also highlights the large magnetic anisotropy of $\mathrm{CeAu}_{0.92} \mathrm{Bi}_{1.6}$. It is noteworthy that the sample presents a broad spin-flop transition $(\approx 10 \mathrm{kOe})$ suggesting that the stoichiometric defects introduce substantial disorder in the system. The solid lines through the data points in Figs. 1(a)-1(c) represent the best fits using a CEF mean field model discussed in detail below.

Figure 2(a) presents the total specific heat divided by temperature $C(T) / T$ as a function of temperature for $\mathrm{CeAu}_{0.92} \mathrm{Bi}_{1.6}$. The peak of $C(T) / T$ defines $T_{\mathrm{N}}=12 \mathrm{~K}$ consistently with the AFM transition temperature observed in the magnetization measurements. We have chosen not to perform detailed analyses of the heat capacity data because
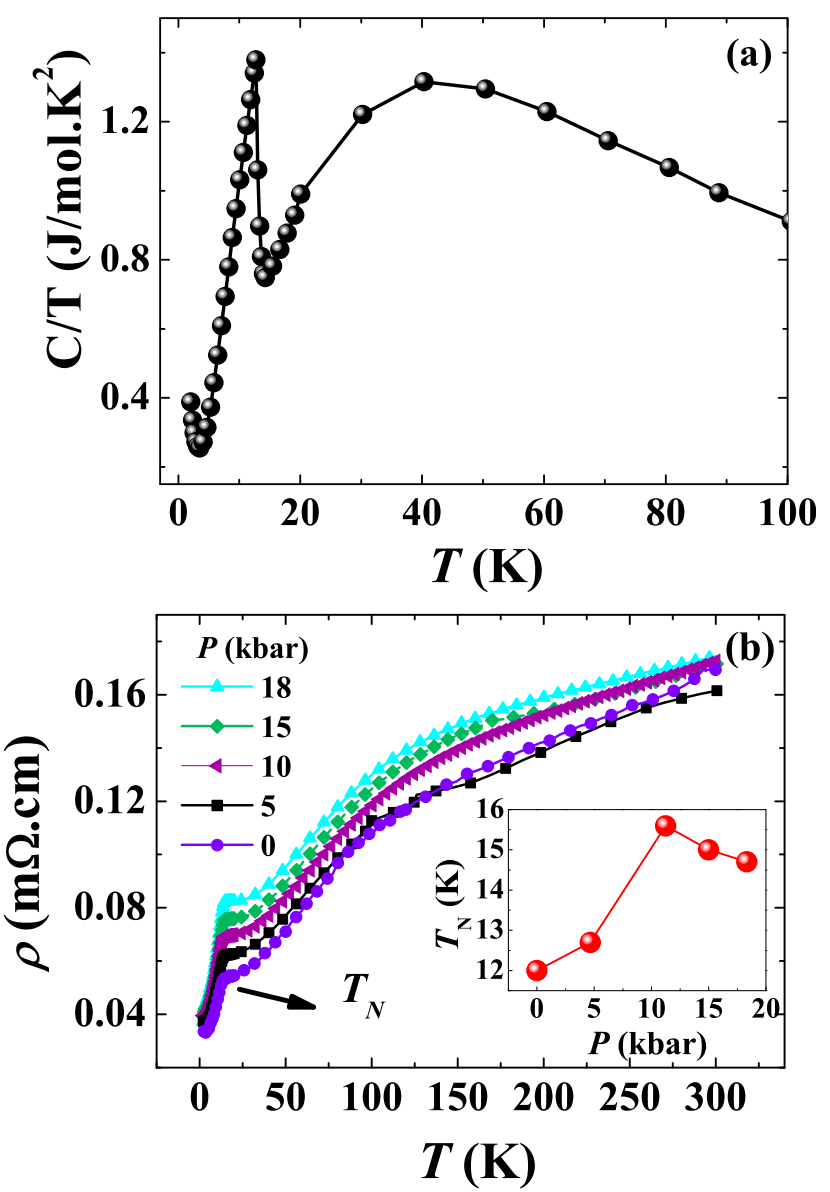

FIG. 2. (a) Total specific heat divided by the temperature of $\mathrm{Ce} \mathrm{Au}_{0.92} \mathrm{Bi}_{1.6}$ as a function of the temperature. (b) Temperature dependence of the electrical resistivity for different values of applied hydrostatic pressure up to 18 kbar. The inset presents the variation of $T_{\mathrm{N}}$ as a function of the pressure. 
we were not able to synthesize a non-magnetic reference compound to properly subtract the lattice contribution of the total specific heat measurements. Since the La-sample does not grow and the Ce-sample is stoichiometric deficient, it is evident that the initial stoichiometry and thermal treatment used here to grow $\mathrm{CeAuBi}$ and $\mathrm{LaAuBi}_{2}$ are not the optimal growth route for these compounds. As such, more attempts are necessary in order to improve the synthesis route for these compounds.

As applied pressure is well known to favor the Kondo effect with respect to the RKKY interaction in Ce-based HF, we now turn our attention to pressure dependent electrical resistivity measurements in order to further investigate the presence of Kondo lattice behavior in $\mathrm{CeAu}_{0.92} \mathrm{Bi}_{1.6 \cdot}{ }^{1-3}$ The in-plane electrical resistivity data $\rho(T, P)$ of $\mathrm{CeAu}_{0.92} \mathrm{Bi}_{1.6}$ are summarized in Fig. 2(b). The electrical resistivity at ambient pressure $\rho(T, P=0)$ shows a broad hump at about $100 \mathrm{~K}$ followed by a sharp drop, as typically found for Ce-based HF. ${ }^{1}$ At lower temperatures, a kink is observed at $T_{\mathrm{N}}=12 \mathrm{~K}$ due to the magnetic ordering. The evolution of the resistivity data as a function of pressure is also presented and two main features can be observed. First, the curvature at $100 \mathrm{~K}$ tends to shift to higher temperatures with increasing pressure. Second, the evolution of the Néel temperature (inset of Fig. 2(b)) shows an initial increase up to $P=10$ kbar with a subsequent decrease up to $18 \mathrm{kbar}$. As the sample presumably presents a high degree of disorder due to the presence of the stoichiometric defects, it is not unreasonable to suppose that the application of pressure can reduce the strain distribution associated with the disorder and, as a consequence, $T_{\mathrm{N}}$ initially increases. Afterward, $T_{\mathrm{N}}$ start decreasing for higher values of applied pressure, suggesting that the main effect is the increase of the Kondo effect. Alternatively, pressure may also initially favor the RKKY interaction and, in turn, increase $T_{\mathrm{N}} \cdot{ }^{1}$ Nevertheless, the slope $\mathrm{d} T_{\mathrm{N}} / \mathrm{d} P$ is relatively small and the slightly increase of the maximum of the resistivity as a function of pressure suggests that the $\mathrm{Ce}^{3+} f$-electrons remain rather localized in the studied pressure range, thus indicating that $\mathrm{CeAu}_{0.92} \mathrm{Bi}_{1.6}$ displays a weak heavy fermion behavior.

In order to understand the evolution of the magnetic properties in the $\mathrm{Ce}_{\mathrm{Bi}}$ family $(T=\mathrm{Cu}$ and $\mathrm{Au}$ ), we have analyzed the data presented in Fig. 1 using a mean field model including the anisotropic interaction from nearestneighbors as well as the tetragonal CEF hamiltonian ${ }^{14}$ for $\mathrm{Ce} \mathrm{Au}_{0.92} \mathrm{Bi}_{1.6}$. This model was used to simultaneously fit $\chi(T)$ and $M(H)$ data, with $T>20 \mathrm{~K}$ as a constrain. The best fits yield the CEF parameters as well as two RKKY exchange parameters, as displayed in Table I. The extracted parameters resulted in a CEF scheme with a $\Gamma_{7}^{(1)}$ ground state doublet $(0.99| \pm 5 / 2\rangle-0.11|\mp 3 / 2\rangle)$, a first excited state $\Gamma_{7}^{(2)}(0.11| \pm 5 / 2\rangle+0.99|\mp 3 / 2\rangle)$ at $189 \mathrm{~K}$ and a second excited doublet $\Gamma_{6}(| \pm 1 / 2\rangle)$ at $283 \mathrm{~K}$. It is interesting to notice that the fits converged only when two distinct $J_{R K K Y}$ exchange parameters were considered, similarly to the $\mathrm{CeCuBi}_{2}$ compound. Despite the fact that both compounds $\left(\mathrm{CeCuBi}_{2}\right.$ and $\left.\mathrm{CeAu}_{0.92} \mathrm{Bi}_{1.6}\right)$ present an $\mathrm{AFM}$ ground state at zero field, the presence of FM fluctuations are evidenced by the presence of a spin-flop transition in the $M(H)$ data. In
TABLE I. Comparison between the extracted parameters (in Kelvin) for $\mathrm{CeCuBi}_{2}$ (Ref. 13) and $\mathrm{CeAu}_{0.92} \mathrm{Bi}_{1.6}$ (this work). Here, $z_{A F M}=2\left(z_{F M}=4\right)$ is the $\mathrm{Ce}^{3+}$ nearest neighbors with an AFM (FM) coupling along the $c$-axis (ab-plane).

\begin{tabular}{lccccc}
\hline \hline & $\mathrm{B}_{2}^{0}$ & $\mathrm{~B}_{4}^{0}$ & $\mathrm{~B}_{4}^{4}$ & $z_{A F M} J_{A F M}$ & ${ }_{F M} J_{F M}$ \\
\hline $\mathrm{CeAu}_{0.92} \mathrm{Bi}_{1.6}$ & -15.57 & 0.01 & 0.76 & 1.4 & -1.1 \\
$\mathrm{CeCuBi}_{2}$ & -7.67 & 0.18 & 0.11 & 1.12 & -1.18 \\
\hline \hline
\end{tabular}

addition, it is important to emphasize that although the obtained CEF scheme can fit the main features of the data shown in Figure 1 (the magnetic anisotropy, and the spinflop transition) to extract the CEF parameters from fits of the macroscopic measurements data may not be unique and/or extremely precise. To directly determine the CEF scheme and its parameters one of the best techniques to employ is inelastic neutron scattering, while X-Ray absorption data can be used to extract the mixed parameters of the wave functions. ${ }^{16}$

Now we are able to compare the obtained CEF scheme for $\mathrm{CeAu}_{0.92} \mathrm{Bi}_{1.6}$ with the one for the parent compound $\mathrm{CeCuBi}_{2}$, also displayed in Table I. Based on this comparison, one can conclude that the RKKY exchange parameters are very similar for both compounds; however, the $\mathrm{B}_{2}^{0}$ parameter is roughly twice larger for $\mathrm{CeAu}_{0.92} \mathrm{Bi}_{1.6}$. The increase of the $\mathrm{B}_{2}^{0}$ value can account for the higher $\chi_{\|} / \chi_{\perp}$ ratio of the magnetic susceptibility data found for the later compound, suggesting that the CEF effects can explain the magnetic anisotropy difference found for the $\mathrm{Ce} T \mathrm{Bi}_{2}$ family. It is worth emphasizing, however, that the unexpected lower Néel temperature value found for $\mathrm{CeAu}_{0.92} \mathrm{Bi}_{1.6}$ can be explained by the presence of disorder in the system, which is known to compete with the magnetic coupling. This effect indicates that higher transition temperatures may be achieved for less disordered samples.

Therefore, the analysis presented here suggests that the $\mathrm{Ce}^{3+} 4 f$ electrons behave as localized magnetic moments for $\mathrm{CeAu}_{0.92} \mathrm{Bi}_{1.6}$. The only indication of a possible heavy fermion behavior is given by the electrical resistivity under pressure measurements. As such, one may speculate that the strong local moment character of the $\mathrm{Ce}^{3+} 4 f$ magnetism in both $\mathrm{CeCuBi}_{2}$ and $\mathrm{CeAu}_{0.92} \mathrm{Bi}_{1.6}$ is a dominant trend in $\mathrm{Ce} T \mathrm{Bi}_{2}$ family ( $T=$ transition metal), which in turn does not favor the emergence of superconductivity in this family.

In fact, the only example of a heavy fermion superconductivity in this family has been reported by a recent work on polycrystalline samples of $\mathrm{CeNi}_{0.8} \mathrm{Bi}_{2},{ }^{11}$ which present an AFM transition at $\sim 5 \mathrm{~K}$ and a superconductor transition at $\sim 4.2 \mathrm{~K}$. The superconducting phase was attributed to the presence of Ni vacancies that would presumably create a different ground state. However, later works have suggested that the superconductivity is more likely to be associated with secondary phases such as $\mathrm{Bi}$ thin films and/or the binaries $\mathrm{NiBi}$ and $\mathrm{NiBi}_{3}{ }^{12,17}$ In particular, besides shedding light on the controversy about the bulk superconductivity in $\mathrm{CeNi}_{0.8} \mathrm{Bi}_{2}$, detailed studies of the physical properties of 
$\mathrm{CeNi}_{x} \mathrm{Bi}_{2-y}$ (for $x=0.62,0.70,0.74$, and 0.78 ) single crystals have also found an enhancement of both the $\mathrm{B}_{2}^{0}$ parameter and the $T_{N}$ as a function of $\mathrm{Ni}$ concentration along the series. ${ }^{17}$ Thus, the evolution of the $\mathrm{Ce}^{3+} \mathrm{CEF}$ scheme causes the doublet ground state to become more isolated from the excited states as one approaches the Ni-rich end. This effect favors the AFM ordering and increases the transition temperature along the series. As such, these results seem to corroborate with the present local moment scenario of the $\mathrm{Ce}^{3+}$ ions in the $\mathrm{Ce} T \mathrm{Bi}_{2}$ family ( $T=$ transition metal).

\section{CONCLUSIONS}

In conclusion, the magnetic properties of $\mathrm{CeAu}_{0.92} \mathrm{Bi}_{1.6}$ are investigated by means of temperature dependent magnetic susceptibility, pressure dependent electrical resistivity, and heat-capacity measurements. Our data reveal that $\mathrm{CeAu}_{0.92} \mathrm{Bi}_{1.6}$ orders antiferromagnetically at $T_{N} \simeq 12 \mathrm{~K}$, although a higher ordering temperature is expected for $\mathrm{Au}-$ stoichiometric samples. The detailed analysis of the macroscopic properties using a mean field model with a tetragonal CEF allows us to obtain the CEF scheme for the studied compound and to understand the evolution of the magnetic anisotropy presented in the $\mathrm{Ce} T \mathrm{Bi}_{2}$ family $(T=\mathrm{Cu}$ and $\mathrm{Au})$. The combined analyses in this investigation suggest that $\mathrm{CeAu}_{0.92} \mathrm{Bi}_{1.6}$ presents a localized $\mathrm{Ce}^{3+} 4 f$ electrons subjected to dominant CEF effects and anisotropic RKKY interactions.

\section{ACKNOWLEDGMENTS}

This work was supported by FAPESP (Grant Nos. 2009/ 09247-3, 2009/10264-0, 2011/01564-0, 2011/23650-5, 2011/
19924-2, 2012/04870-7, 2012/05903-6, 2012/10675-2, and 2013/20181-0), CNPq, and CAPES-Brazil.

${ }^{1}$ A. C. Hewson, The Kondo Problem To Heavy Fermions (Cambridge University Press, Cambridge, 1993).

${ }^{2}$ P. Coleman, C. Pépin, Q. Si, and R. Ramazashvili, J. Phys. Condens. Matter 13, R723-R738 (2001)

${ }^{3}$ J. D. Thompson and Z. Fisk, J. Phys. Soc. Jpn. 81, 011002 (2012)

${ }^{4}$ C. Petrovic et al., J. Phys.: Condens. Matter 13, L337 (2001).

${ }^{5}$ V. A. Sidorov et al., Phys. Rev. Lett. 89, 157004 (2002).

${ }^{6}$ A. Thamizhavel et al., J. Phys. Soc. Jpn. 72, 2632 (2003).

${ }^{7}$ F. Steglich, J. Aarts, C. D. Bredl, W. Lieke, D. Meshede, W. Franz, and H. Schafer, Phys. Rev. Lett. 43, 1892 (1979).

${ }^{8}$ H. Hegger, C. Petrovic, E. G. Moshopoulou, M. F. Hundley, J. L. Sarrao, Z. Fisk, and J. D. Thompson, Phys. Rev. Lett. 84, 4986 (2000).

${ }^{9}$ J. Ye, Y. K. Huang, K. Kadowaki, and T. Matsumoto, Acta Crystallogr. Sect. C 52, 1323 (1996).

${ }^{10}$ M. H. Jung, A. H. Lacerda, and T. Takabatake, Phys. Rev. B 65, 132405 (2002).

${ }^{11}$ H. Mizoguchi, S. Matsuishi, M. Hirano, M. Tachibana, E. TakayamaMuromachi, H. Kawaji, and H. Hosono, Phys. Rev. Lett. 106, 057002 (2011).

${ }^{12}$ X. Lin, W. E. Straszheim, S. L. Budko, and P. C. Canfield, J. Alloys Compd. 554, 304 (2012).

${ }^{13}$ C. Adriano, P. F. S. Rosa, C. B. R. Jesus, J. R. L. Madergan, T. M. Garitezi, T. Grant, Z. Fisk, D. J. Garcia, A. P. Reyes, P. L. Kuhns, R. R. Urbano, C. Giles, and P. G. Pagliuso, Phys. Rev. B 90, 235120 (2014).

${ }^{14}$ P. G. Pagliuso, D. J. Garcia, E. Miranda, E. Granado, R. Lora Serrano, C. Giles, J. G. S. Duque, R. R. Urbano, C. Rettori, J. D. Thompson, M. F. Hundley, and J. L. Sarrao, J. Appl. Phys. 99, 08 P703 (2006).

${ }^{15}$ C. B. R. Jesus, M. M. Piva, P. F. S. Rosa, C. Adriano, and P. G. Pagliuso, J. Appl. Phys. 115, 17E115 (2014).

${ }^{16}$ T. Willers, Z. Hu, N. Hollmann, P. O. Körner, J. Gegner, T. Burnus, H. Fujiwara, A. Tanaka, D. Schmitz, H. H. Hsieh, H.-J. Lin, C. T. Chen, E. D. Bauer, J. L. Sarrao, E. Goremychkin, M. Koza, L. H. Tjeng, and A. Severing, Phys. Rev. B 81, 195114 (2010).

${ }^{17}$ P. F. S. Rosa, C. B. R. Jesus, C. Adriano, Z. Fisk, and P. G. Pagliuso, "Role of $\mathrm{Ni}$ vacancies on the physical properties of $\mathrm{CeNi}_{1-\mathrm{x}} \mathrm{Bi}_{2}$ single crystals," J. Phys. Conf. Ser. (submitted). 